\title{
The Study on the Influence of Workplace Gossip on Employees' Work Enthusiasm
}

\author{
Liang Yan ${ }^{1, a}$, Qian Zhang ${ }^{2, b^{*}}$ \\ ${ }^{1}$ International Business School Shaanxi Normal University Xi'an, Shaanxi \\ ${ }^{2}$ International Business School Shaanxi Normal University Xi'an, Shaanxi
}

\begin{abstract}
The impact of workplace gossip on employees has become a hot topic among researchers and practitioners. The research integrates both positive and negative dimensions, and more comprehensively explores the influence of workplace gossip on employees' work enthusiasm to make theoretical contributions to workplace gossip research. Based on the valid sample of 201 employees from various types of enterprises in Xi' an, the study uses SPSS 21.0 and AMOS 24.0 software to conduct data analysis and test the research hypothesis. The empirical results show that positive gossip in the workplace has a significant positive impact on employees' work enthusiasm, and negative workplace gossip has a significant negative impact on employees' work enthusiasm. Finally, according to the research results, this article puts forward corresponding management improvement strategies and suggestions.
\end{abstract}

\section{INTRODUCTION}

The phenomenon of gossip is widespread and even popular in real life, and the workplace, as the place where people live the longest time outside the family, naturally becomes part of the gossip dissemination place, and the workplace gossip has also become the main informal information dissemination channel within the organization. The workplace gossip, as the name suggests, is the spread of gossip that occurs in the workplace, in the organization, and between employees. As a type of social gossip, workplace gossip, like social gossip, has characteristics that cannot be completely eliminated.

The research on workplace gossip in the West has been developing, and the research scope has gradually expanded to the dissemination motivation, models, relevant influencing factors and other aspects [1]. However, there are only a few studies on workplace gossip in China, and the content is mainly focused on the results of negative gossip. The idea of "human words are awesome" contained in traditional Chinese thinking also makes managers think that workplace gossip brings negative effects. However, with the deepening of research, it is found that workplace gossip not only has negative effects, but also has positive effects [2]. Therefore, a comprehensive discussion of the positive and negative effects of workplace gossip is of great significance to related theories and practices.

Work enthusiasm refers to the emotional, dynamic and conscious psychological tendency shown by employees through their behaviors under the interaction of their internal needs, external motivation and goals, as well as the mental state of their active efforts in the process of

ae-mail: vivi8235@163.com

bCorresponding author's e-mail: zq15091598781@163.com realizing their goals [3]. Good work enthusiasm provides a strong internal driving force for improving work performance. But so far, no scholar has studied the influence of workplace gossip on employees' work motivation. Although relevant theoretical analysis has been carried out, there is no empirical data to support relevant theories and conclusions. Based on the above background, this article attempts to explore the influence relationship between workplace gossip and employee work enthusiasm in order to make theoretical contributions.

\section{THEORY AND RESEARCH HYPOTHESIS}

\subsection{Definition and dimensions of workplace gossip}

The term gossip entered people's lives a long time ago, and its concept first refers to chats between relatives and friends [4]. With the deepening of research, scholars have put forward a comprehensive and detailed definition of gossip based on the scientific nature of the research. On the basis of comprehensive consideration of the characteristics and nature of gossip and drawing on previous studies, Foster believes that gossip is the behavior of disseminating private information of an absent third party with personal evaluation between two or more people in a certain relevant situation [5].

Scholars have their own understanding and expression of the concept of workplace gossip. Among them, Foster's definition of gossip is more respected. This definition covers the subject of the gossip (the communicator, the receiver, the non-present gossip target), the nature of the 
content (evaluative), and the environmental characteristics (under certain circumstances) of the gossip. It clearly and comprehensively defines the gossip. Scholars in the research have also elaborated on the concept of gossip, but they are basically similar to this concept.

After understanding the definition and characteristics of gossip, it is not difficult to understand workplace gossip. By summarizing the relevant literature and scholars' definitions of workplace gossip, this article believes that workplace gossip is an informal, evaluative conversation conducted by employees in an organization against an employee who is not present in the organization. The content of the conversation can be related to work. Relevant or not related to work. And workplace gossip, as a kind of gossip, has the same nature and characteristics as gossip.

In terms of the dimensions of workplace gossip, classification based on the content valence of gossip is a common classification method. According to whether the information exchange about the absent party is constructive or destructive, and whether it is praise or accusation, the gossip is classified as positive, negative or neutral according to the content valence [6]. Foster has shown in research that both positive and negative gossip have value in the workplace. He believes that gossip will definitely affect reputation. However, there is no evidence that it must be caused by negative gossip, and it may not necessarily be a negative influence [5]. In other words, in addition to negative gossip, positive gossip may also have an important impact on employees. Therefore, it is necessary to distinguish the content and valence of gossip and to classify and research it.

\subsection{Definition of work motivation}

Gubman once put forward the definition of work enthusiasm in his research: the individual has a clear understanding of the organization's goals, has the courage to overcome difficulties when completing the work, and dares to accept challenges and changing emotions [7]. If a person with weaker abilities has a strong motivation to work, he is willing to improve his abilities and is willing to make more efforts to improve his work abilities. Gradually, with his own enthusiasm for work, he constantly overcomes difficulties and breaks through his own limitations, so as to improve his ability and obtain better performance output. On the contrary, if a person with outstanding work ability lacks the enthusiasm and enthusiasm of the work, even in the field and work he is good at, he will be passive.

Domestic scholars are also aware of the work enthusiasm for the importance of the organization and management, Feng Jiangping and Li Yuanyuan in research on China's new generation staff's work enthusiasm, to define motivation as "workers, in needs, motivation, goal, under the interaction of through the behavior of a kind of consciousness, the dynamic and exciting psychological tendency, and in the process of target hard up state of mind" [3]. This definition points out the influencing factors, mechanism and results of work enthusiasm.

\subsection{Positive gossip and work motivation in the workplace}

According to the social comparison theory [6], employees will naturally compare themselves with the gossip content in the process of participating in workplace gossip. For example, when employees find that the work behavior or performance of a gossip goal is recognized and praised by everyone, they will compare their behavior and performance with the gossip goal and find the gaps and shortcomings. In order to keep their behavioral performance consistent with the Gossip goal, or even exceed him, employees will have a strong motivation to improve their behavior, improve their performance, and generate strong work enthusiasm. Wert and Salovey pointed out in their research that the motivation for social comparison is not only the need for self-evaluation, but also self-improvement and self-improvement. They believe that gossip, especially positive gossip about others who are better than themselves, can promote selfimprovement [8]. According to the social comparison theory, Grosser's research shows that there is a positive correlation between the degree of a person's participation in positive gossip and the performance evaluated by the boss [6].

In summary, the study puts forward hypothesis 1:

Hypothesis 1: Positive gossip in the workplace has a significant positive impact on employees' work motivation.

\subsection{Negative gossip and work motivation in the workplace}

According to the content of social comparison theory, social comparison theory includes upward comparison theory and downward comparison theory. Among them, the downward comparison theory is to compare with others who are inferior to oneself [9]. When negative gossip is rampant in the workplace in a certain organization, employees often receive relatively negative information from others, which keeps employees in a downward comparative environment for a long time. The downward social comparison will have an emotional impact, and a related emotion is pride. When people compare themselves with others and find that their current situation is superior to others, they may feel proud if they think that their own advantages are due to their own internal advantages or characteristics [6]. Moreover, a long-term downward comparison will make employees too optimistic about their current situation, and even lead to employees' overconfidence [10]. This sense of pride and overconfidence will further make employees feel superior, thereby reducing work initiative and enthusiasm.

In summary, the study puts forward hypothesis 2 :

Hypothesis 2: Negative gossip in the workplace has a significant negative impact on employees' work motivation. 


\section{RESEARCH METHODS}

\subsection{Research sample}

In this study, employees of various enterprises in Xi'an were selected as survey subjects, and data were collected through various online and offline survey methods. In the end, a total of 223 questionnaires were collected online and offline, of which 201 were valid questionnaires, with an effective rate of $90.13 \%$. The sample structure is shown in Table I.

\subsection{Research tools}

\subsubsection{Workplace Gossip Scale}

TABle I SAMPLE STRUCTURE $(\mathrm{N}=201)$

\begin{tabular}{|c|c|c|c|c|c|}
\hline Cate-gory & Feature & Propor-tion & Cate-gory & Feature & Propor-tion \\
\hline \multirow{4}{*}{ Age } & $\leqslant 25$ & $50.75 \%$ & \multirow{2}{*}{ Gender } & Male & $44.28 \%$ \\
\hline & $26-35$ & $35.32 \%$ & & Female & $55.72 \%$ \\
\hline & $36-45$ & $10.95 \%$ & \multirow{5}{*}{$\begin{array}{c}\text { Workin-g } \\
\text { years }\end{array}$} & $\leqslant 5$ years & $62.19 \%$ \\
\hline & $\geqslant 46$ & $2.99 \%$ & & $6-10$ years & $20.9 \%$ \\
\hline \multirow{7}{*}{$\begin{array}{c}\text { Type of } \\
\text { enterp-rise }\end{array}$} & Service class & $29.85 \%$ & & $11-15$ years & $7.46 \%$ \\
\hline & Education & $17.41 \%$ & & $15-20$ years & $4.98 \%$ \\
\hline & Technology & $19.9 \%$ & & $\geqslant 21$ years & $4.48 \%$ \\
\hline & Manufactu-ring & $11.94 \%$ & \multirow{7}{*}{ Type of jobs } & Transactio-nal & $21.89 \%$ \\
\hline & Career & $12.94 \%$ & & Skilled & $31.34 \%$ \\
\hline & Constructi-on & $1.49 \%$ & & Artistic & $7.96 \%$ \\
\hline & Other & $6.47 \%$ & & Social & $8.96 \%$ \\
\hline \multirow{3}{*}{ Positi-on level } & Grassroots & $72.14 \%$ & & Research & $7.46 \%$ \\
\hline & Middle level & $12.89 \%$ & & Managem-ent & $13.93 \%$ \\
\hline & High-level & $5.97 \%$ & & Other & $8.46 \%$ \\
\hline
\end{tabular}

\subsubsection{Work motivation scale}

The work motivation scale draws on the research scales of Feng Jiangping, Li Yuanyuan, Chen Hong, and Zhang Yue [3]. The scale has a total of 24 items, such as: I will continue to summarize and reflect, and continue to improve in my work. Among the options, 1 means strongly agree, 2 means agree, 3 means disagree, and 4 means strongly disagree. The internal consistency reliability of the employee work motivation scale in this study is 0.977 .

\section{DATA ANALYSIS AND RESULTS}

\subsection{Reliability analysis}

The research passed SPSS 21.0 to test the reliability of the scale (Cronbach's alpha), and the test results are shown in Table 3. The results show that the Alpha coefficient of the workplace gossip scale is 0.907 , of which the alpha coefficient of the positive gossip part of the workplace is 0.909 , and the alpha coefficient of the negative gossip part
The Workplace Gossip Scale is derived from a scale developed by Kuo, Chang, Quinton, Lu and Lee on the Workplace Gossip Scale [11]. The scale originally used Likert's five-point scoring method, which was revised to a four-point scoring method in order to be consistent with the employee's work motivation measurement scale. The questions covered by the scale are mainly divided into two parts: positive workplace gossip and negative workplace gossip, such as "colleagues recent happy life events, buying a house or car" and "colleagues recent miserable life events, disease or car accident". The scale includes a total of two dimensions, each with 10 items, a total of 20 items. Among the options, 1 means always, 2 means often, 3 means occasionally, and 4 means never. The internal consistency reliability of the Workplace Gossip Scale in this study is 0.907 . of the workplace is 0.961 . The Alpha coefficient of the employee motivation scale is 0.977 , which are both greater than 0.800 , indicating that the scales have good internal consistency.

\subsection{Validity analysis}

The scales used in this article are mature scales selected after a lot of reference to relevant research and literature, and each scale has passed the empirical test of scholars. Therefore, this paper adopts confirmatory factor analysis method to test the validity of this research questionnaire.

Using AMOS 24.0 software, according to the valid data collected in this study, the aggregate validity of the questionnaire model was tested. The analysis results are shown in Table II with the three-factor model data. According to the analysis data in Table II, RMSEA is less than 0.08 , NNFI is greater than $0.90, \mathrm{CFI}$ is greater than $0.90, \mathrm{X} 2 / \mathrm{DF}=1.983$, less than 5 , indicating that the model has good aggregation validity.

In this study, a confirmatory factor analysis model was used for nested comparison to analyze the discriminative validity between variables. According to the design method of the measurement indicators in the confirmatory factor analysis, the three variables involved in this study 
were regrouped and merged. Three measurement models were created according to different merging methods, and the comparison results are shown in Table II. According to the comparison results in Table II, the fit indexes of the three-factor model used in this study are better than other nested models, and the chi-square test results also show that the three-factor model is better. Therefore, it shows that the three-factor model used in this study has good discriminative validity among the variables. used to test the correlation between these three variables. The results are shown in Table III. The results show that there is a significant positive correlation between positive workplace gossip and employees' work enthusiasm, and there is a significant negative correlation between workplace negative gossip and employees' work enthusiasm.

\subsection{Correlation analysis}

This study intends to explore the impact of positive workplace gossip and negative workplace gossip on employees' work motivation, so SPSS 21.0 software was

\begin{tabular}{|c|c|c|c|c|c|c|}
\hline $\begin{array}{l}\text { Measure-ment } \\
\text { model }\end{array}$ & $\begin{array}{l}\text { Model inclusion } \\
\text { factor }\end{array}$ & $\mathrm{X}^{2} / \mathbf{d f}$ & RMS-EA & NN-FI & CFI & $\triangle \mathrm{X}^{2}$ \\
\hline Single factor & $\begin{array}{c}\text { PWG+NGW+ } \\
\text { EWE }\end{array}$ & 4.751 & 0.137 & 0.63 & 0.67 & \\
\hline Two-factor & PWG+NGW, EWE & 3.229 & 0.106 & 0.78 & 0.804 & $1286.607 * *$ \\
\hline Three factors & PWG, NGW, EWE & 1.983 & 0.07 & 0.903 & 0.919 & $1158.752 * *$ \\
\hline
\end{tabular}

TABLE III CORRELATION ANALYSIS

\begin{tabular}{|c|c|c|c|}
\hline variable & $\begin{array}{c}\text { Positive workplace } \\
\text { gossip }\end{array}$ & $\begin{array}{c}\text { Negative workplace } \\
\text { gossip }\end{array}$ & Work motivation \\
\hline $\begin{array}{c}\text { Positive workplace } \\
\text { gossip }\end{array}$ & 0.909 & 0.961 & \\
\hline $\begin{array}{c}\text { Negative workplace } \\
\text { gossip }\end{array}$ & 0.119 & $-0.429 * *$ & 0.977 \\
\hline Work motivation & $0.218^{* *}$ & & \\
\hline
\end{tabular}

Note: $* *$ means $\mathrm{p}<0.01$, and the correlation is significant. The diagonal is the alpha coefficient

\subsection{Regression analysis}

According to the results of the correlation analysis, further linear regression analysis was performed on the data. The analysis results are shown in Table IV. The results show that compared with model 1 , model 2 and model 3 have improved R2, indicating that both model 2 and model 3 can explain workplace gossip. And when the position level of the control variable is significant, the regression coefficient of positive gossip in the variable workplace $\beta=0.208^{* *}, p<0.01$, and the regression coefficient of negative gossip in variable workplace $\beta=-0.392 * * *$, $\mathrm{p}<0.001$, both are shown significantly. Therefore, Hypothesis 1 and Hypothesis 2 of this study are both valid.

TABLE IV REGRESSION ANALYSIS

\begin{tabular}{|c|c|c|c|c|}
\hline & & \multicolumn{3}{|c|}{ Standardization factor } \\
\hline & & Model 1 & Model 2 & Model 3 \\
\hline \multirow{4}{*}{$\begin{array}{c}\text { Control } \\
\text { variable }\end{array}$} & Gender & 0.052 & 0.039 & 0.079 \\
\cline { 2 - 5 } & Age & -0.113 & -0.133 & -0.079 \\
\cline { 2 - 5 } & Working years & -0.008 & -0.010 & -0.016 \\
\cline { 2 - 5 } & Type of jobs & 0.101 & 0.104 & 0.068 \\
\cline { 2 - 5 } & Type of enterprise & -0.001 & -0.007 & -0.005 \\
\hline \multirow{2}{*}{$\begin{array}{c}\text { Independent } \\
\text { variable }\end{array}$} & Position level & $0.297 * * *$ & $0.283^{* * *}$ & $0.249^{* *}$ \\
\hline \multirow{3}{*}{\begin{tabular}{c} 
Model fitting \\
\cline { 2 - 5 }
\end{tabular}} & Positive gossip & & $0.208^{* *}$ & $-0.392^{* * *}$ \\
\cline { 2 - 5 } & Negative gossip & & & 0.291 \\
\cline { 2 - 5 } & $\mathrm{R} 2$ & 0.141 & 0.184 & 0.266 \\
\hline
\end{tabular}

Note: ${ }^{\mathrm{p}}<0.05,{ }^{* *} \mathrm{p}<0.01,{ }^{* * *} \mathrm{p}<0.001$

\section{ConClusion AND DISCUSSION}

The research focuses on the relationship between positive workplace gossip and workplace negative gossip and employees' work enthusiasm. Empirical research is carried out and the following conclusions are obtained: (1) Positive workplace gossip has a significant positive impact on employees' work motivation; (2) Negative workplace gossip has a significant negative impact on 
employees' work motivation.

According to the findings of this study, positive workplace gossip will have a positive impact on employees' work motivation. As managers, they should be aware of the advantages of informal information dissemination channels, and use informal information dissemination to go to the grassroots level to understand employees' psychology and work status. Then managers can use formal channels to create positive workplace gossip themes, thereby establishing a positive organizational culture. For example, when an employee has strong work ability and outstanding work performance, he can be publicly praised on appropriate occasions. Organizations can regularly hold commendation meetings to reward employees with outstanding performance this year or this quarter, and hold experience sharing meetings to allow outstanding employees to share their work experience with other employees. By creating and actively disseminating positive gossip, promote positive work status and ideas, and encourage employees to learn. Through this behavior, the praised employees can also gain a strong self-identity and organizational recognition, so that all employees can realize that the organization recognizes their work, thereby enhancing their confidence in the organization.

According to the results of this study, negative workplace gossip will have a negative impact on employees' work motivation. In order to avoid the adverse effects caused by negative gossip in the workplace, on the one hand, the organization can properly establish relevant rules and regulations, on the other hand, it should provide employees with a good communication environment and establish a positive corporate culture. Because when negative gossip occurs in the workplace, in order to avoid further negative effects, measures should be taken to stop the rumors in a timely manner, and the best way to stop negative gossip in the workplace is to communicate in good faith. For individuals, in order to prevent themselves from continuing to be attacked by negative gossip in the workplace, they should communicate with gossip communicators in time to eliminate misunderstandings. For the organization, an open system should be established as much as possible, so that employees can be more aware of the current situation of the organization and related policies, rather than guessing. For employees who frequently spread negative gossip in the workplace, managers should keep abreast of the employee's psychology and state, and communicate and enlighten them.

When communicating, informal communication can make employees put down their guard and send more information. Therefore, it is required to form a good informal communication atmosphere in the organization to create a good environment for sincere communication between employees, so that employees can communicate relatively relaxed and freely. At the same time, encourage active and sincere communication between employees and between employees and managers, and finally eliminate misunderstandings through communication. Specific implementation suggestions include that organizations can create informal communication environments such as tea rooms, managers can hold informal meetings such as round table discussions, and other feasible informal communication methods.

There are still many points in the article that can be further advanced in future research to improve the research conclusions. First, this study did not study the dimensions of this variable when discussing the work enthusiasm of employees. If you want to get more specific research results, you should also divide the dimensions and study them separately. Second, this article does not consider the influence of intermediate variables and moderating variables. In subsequent related research, the influence of intermediate variables such as personality characteristics and organizational culture can be considered to make the model richer and more reasonable. Third, this study uses a foreign maturity scale, and the results may be affected due to cultural differences. Therefore, future research can further develop the workplace gossip scale in the Chinese context. Through the above methods, research can continue to deepen and improve the relationship between workplace gossip and employee work enthusiasm.

\section{REFERENCES}

1. Sun Huijie, Mai Xiaoqin. Workplace Gossip: Functions and Impact Factors[J]. China Human Resources Development, 2016, (21):45-70.

2. Chen Xingwang. Research on the Causes and Effects of Negative Gossip Behavior in Workplace[J].Modern Communication, 2016:128129.

3. Feng Jiangping, Li Yuanyuan, Chen Hong, Zhang Yue. The measurement research of the new generation of employees' work motivation[J]. Journal of Yunnan Normal University (Philosophy and Social Sciences Edition), 2013:58-66.

4. Chen Lijun, Cai Yamian. Discussion on the effect of gossip behavior in the workplace[J]. Journal of Hubei Second Normal University, 2017:80-84.

5. Foster E K. Research on gossip: Taxonomy, methods, and future directions[J]. Review of General Psychology, 2004, 8(2): 78-99.

6. Travis J. Grosser, Virginie Lopez-Kidwell, Giuseppe Labianca. A Social Network Analysis of Positive and Negative Gossip in Organizational Life [J]. Group \& Organization Management, 2010, 35(2):177-212.

7. Gubman, Ed. From engagement to passion for work: the search for the missing person[J].Human Resource Planning,2004,(27):42-60.

8. Sarah R. Wert, Peter Salovey. A social comparison account of gossip[J].Review of General Psychology,2004,8(2),122-137.

9. Zhang Bao, Kong Fanlan, Zhao Yufang. Overview of Social Comparative Research[J]. Science \& Technology Information, 2008, (31):340-390.

10. Liu Bai. Comparison of corporate performance and overconfidence of managers [J]. Finance and Economics, 2020, (1): 65-47. 
11. Kuo, Chang, Quinton, Lu, Lee. Gossip in the workplace and the implications for hr management: A study of gossip and its relationship to employee
cynicism[J]. International Journal of Human

Resource Management, 2014, 26(18): 1-20. 